IUHET-405

March 1999

\title{
Leptogenesis and Yukawa textures
}

\author{
Micheal S. Berger * and Biswajoy Brahmachari ${ }^{\dagger}$ \\ Physics Department, Indiana University, Bloomington, IN 47405, USA
}

\begin{abstract}
We study a set of textures giving rise to correct masses and mixings of the charged fermions in the context of leptogenesis. The Dirac neutrino texture pattern is assumed to be identical with the up quark texture. The heavy Majonana neutrino mass matrix is obtained by inverting the type-I see-saw formula and using the neutrino masses and mixings required by the solar and atmospheric neutrino oscillation experiments as input. After making the feasibility study of the generated lepton asymmetry via the decay of the heavy right handed neutrino, we compute the generated baryon asymmetry by numerically solving the supersymmetric Boltzmann equations. We find for these models that both the hierarchy of the texture as well as the placement of the texture zeros are important to the viability of leptogenesis as the source of the observed baryon asymmetry of the universe.
\end{abstract}

\footnotetext{
*Electronic address: berger@gluon.physics.indiana.edu

${ }^{\dagger}$ Electronic address: biswa@gluon2.physics.indiana.edu
} 


\section{INTRODUCTION}

Relationships between fermion masses and mixings have been the subjects of much theoretical interest starting with a postulated relationship between the Cabibbo angle and the down and strange quark masses. Most of the unknown parameters in the Standard Model (SM) occur in the Yukawa sector and any relationships between these parameters is welcome theoretically and experimentally testable. The interest in models of fermion masses and mixings accelerated with the advent of grand unified theories. In these models, the gauge multiplets of the Standard Model are unified into multiplets of the grand unified gauge group, and relationships between the parameters emerge naturally as a consequence of the larger symmetry. These models can be augmented by global symmetries or texture zeros in the Yukawa coupling matrices can be assumed, leading to further predictions.

A mystery of the Yukawa sector is the obvious hierarchy that exists in fermion masses and mixings. The top quark mass is much larger than the charm quark mass which is still much larger than the up quark mass, for example. The (Cabbibo-Kobayashi-Maskawa) CKM matrix is measured experimentally have small mixing angles. Clearly a fundamental theory that explains the origin of the couplings in the Yukawa sector rather than just parameterizing them, should explain these features. The Yukawa sector of the Standard Model is parametrized in terms of $3 \times 3$ matrices, so the hierarchy exhibits itself as a hierarchy among the elements of these matrices.

The usual predictions from these models of fermion masses and mixings are relationships between the masses of quarks and leptons or between the mixing angles of the CKM matrix and dimensionless ratios of the quark and lepton masses. In addition there are often predictions for the amount of charge conjugation-parity $(\mathrm{CP})$ violation and predictions for the CP asymmetries of meson decays. It is of interest to consider whether further constraints are obtained after making reasonable assumptions about some other physical observable. In this paper we consider textures (or patterns of zero entries) of Yukawa coupling matrices and assume that the baryon asymmetry has its origins in the decay of heavy Majorana neutrinos 
which violate lepton number. The asymmetry in lepton number is recycled into a baryon number asymmetry via the sphaleron process. This idea was first put forward by Fukugita and Yanagida [1] and has come to be known as baryogenesis via leptogenesis. While this constraint is admittedly more speculative than the comparison of masses and mixing angles derived from experiment, it is instructive to determine which properties of the Yukawa textures are essential for the baryogenesis via leptogenesis to work.

Sakharov pointed out [2] that a small baryon asymmetry may have been produced in the early universe if three conditions are satisfied: 1) baryon number is violated, 2) charge conjugation symmetry (C) and $\mathrm{CP}$ are violated, and 3) there is a departure from thermal

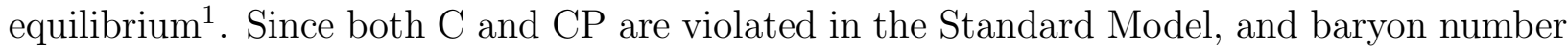
is violated by a nonperturbative effect called sphalerons, the natural place to look first to explain the generation of a baryon asymmetry is within the Standard Model itself. However this line reasoning does not work: the required Higgs masses is too small and has been ruled out by the direct searches at LEP [4]. One can try to extend the Standard Model: one popular attack is to consider the Minimal Supersymmetric Standard Model (MSSM) and assume that the source of the CP violation is still contained within the CKM matrix [5]. One can achieve the observed baryon asymmetry, but only at the expense of going to a corner of parameter space, namely one requires a light scalar top quark (top squark or stop). This kind of solution should rightly be regarded as unnatural on theoretical grounds, although it is of much experimental and phenomenological interest primarily because it is just out of reach.

We pursue in this paper the alternative approach of baryogenesis via leptogenesis: With the confirmation of the atmospheric neutrino anomaly in the SuperKamiokonde [6], it seems plausible to start from the point of view that it is likely that the neutrino mixing is occuring

\footnotetext{
${ }^{1} \mathrm{~A}$ baryon asymmetry could arise even in thermal equilibrium if CPT is violated. See, for example, Ref. [3].
} 
and the neutrinos have masses given by a see-saw mechanism. The heavy Majorana neutrino mass matrix is obtained by inverting the type-I see-saw formula

$$
m_{\mathrm{eff}}=m_{D}^{T} \frac{1}{M_{N}} m_{D}
$$

and using the neutrino masses and mixings required by the solar and atmospheric neutrino oscillation experiments as inputf. The neutrino sector contains a new source of CP violation; the interference between tree-level and one-loop contributions to the Majorana neutrino decays can give rise to a lepton asymmetry. In this scenario, the amount of $\mathrm{CP}$ violation that gives rise to a lepton asymmetry and ultimately to a baryon asymmetry depends critically on the Dirac mass matrix of the neutrinos in two ways: (1) the overall hierarchy pattern of the matrix and (2) the placement of the texture zeros]. Furthermore, the generated lepton asymmetry can be erased by subsequent lepton-number violating scattering and this dilution can depend on the placement of the texture zeros.

In this paper we start from the position that the positive observation in the solar and atmospheric neutrino experiments suggest that there is a new scale of heavy physics. We assume that heavy right-handed neutrinos exist and the lightness of the observed neutrinos is the result of a seesaw mechanism. In this framework we study the plausibility of leptogenesis in the case of neutrino Dirac mass matrices with texture zeros and hierarchical structure similar to the ones that are consistent with low-energy data in the quark sector.

\section{YUKAWA TEXTURES}

\footnotetext{
${ }^{2}$ We neglect the possibility that there are contributions from a left-handed triplet Higgs

${ }^{3}$ If a texture zero predicts a small level of $\mathrm{CP}$ violation at the GUT scale, this suppression will be preserved by the renormalization group scaling.
} 
TABLES

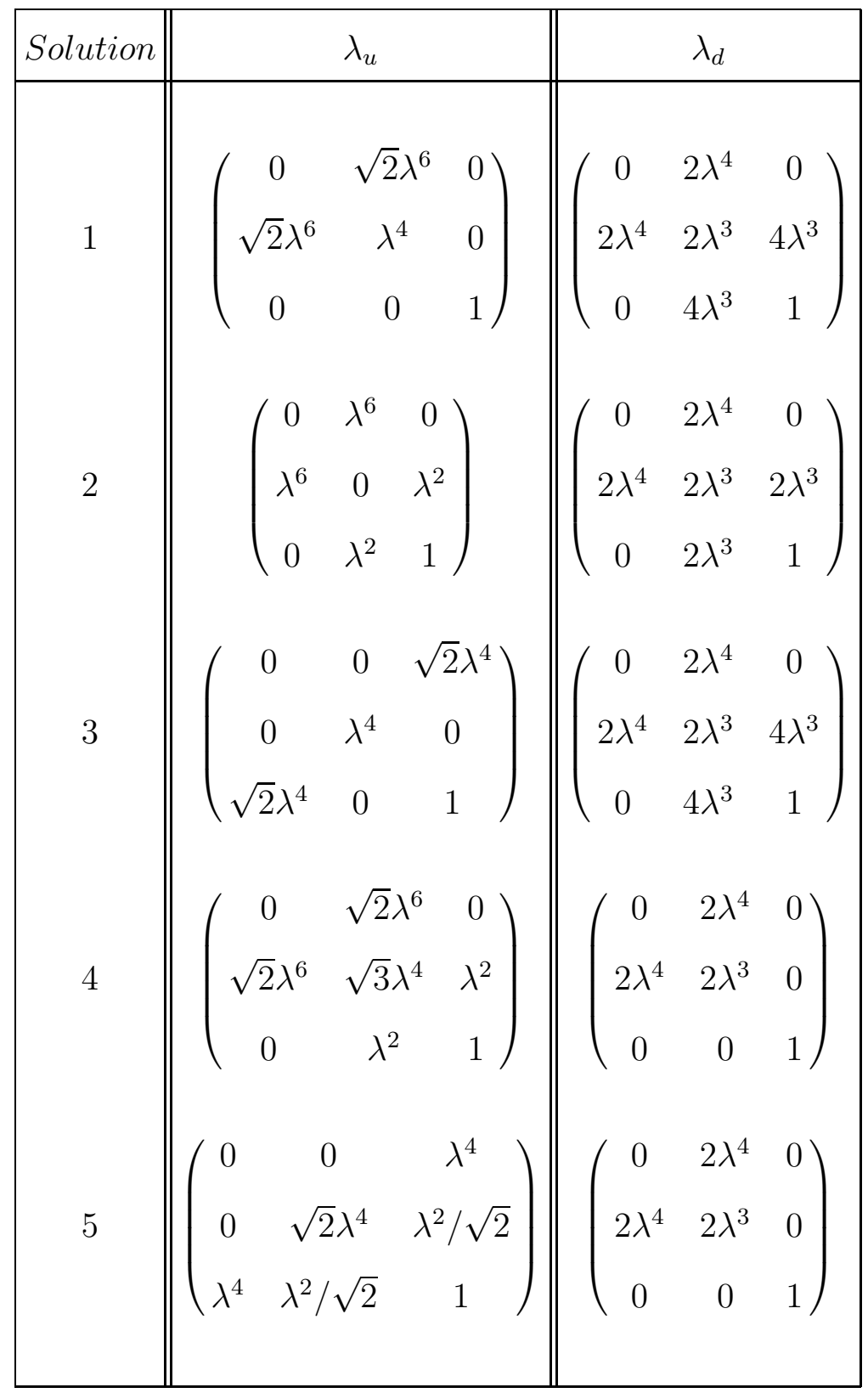

TABLE I. The list of textures suggested by RRR. 
Ramond, Ross and Roberts (RRR) performed [8] a systematic search for all possible symmetric quark and lepton mass matrices with five texture zeros at the unification scale that are compatible with low-energy measurements. They found a total of five possible solutions, which we display again in Table —for convenience.

We assume the Dirac neutrino mass matrix at the GUT scale has the same texture zeros as the up quark matrix

$$
m_{D}^{\prime} \simeq m_{u}=\lambda_{u} v \sin \beta .
$$

In certain situations where the Yukawa interactions are minimal, grand unified symmetry enforces an exact equality. More generally one might expect the equality of elements in the neutrino texture and the up quark texture not be exact, but be related by Clebsch coefficients (very often 3). These Clebsch factors are typically small and do not upset the hierarchy of the matrices. The general qualitative features of our analysis is not affected by these factors of order one, since the amount of baryon asymmetry generated in a model with a particular texture is governed by the hierarchy (given in terms of the parameter $\lambda$ which is fixed by the Cabibbo angle) and the position of the texture zeros.

\section{NUMERICAL SOLUTIONS}

We consider small angle MSW solution of the solar neutrino problem through the mixing $\nu_{e} \leftrightarrow \nu_{\mu}$ and maximal mixing solution of atmospheric neutrino oscillation through the mixing

$\nu_{\mu} \leftrightarrow \nu_{\tau}$. We take as inputs the following neutrino masses consistent with the experimental measurements, namely [0, [15]

$$
\begin{gathered}
0.8<\sin ^{2} 2 \theta_{23}<1, \quad 10^{-3}<\Delta m_{23}^{2}<10^{-2}, \\
3 \times 10^{-3}<\sin ^{2} 2 \theta_{12}<2 \times 10^{-2}, \quad 5 \times 10^{-6}<\Delta m_{12}^{2}<10^{-5} .
\end{gathered}
$$

The inverse neutrino mass matrix is 


$$
m_{\mathrm{eff}}^{-1}=\left(\begin{array}{ccc}
1 / m_{1} & 0 & 0 \\
0 & 1 / m_{2} & 0 \\
0 & 0 & 1 / m_{3}
\end{array}\right)
$$

This can be rotated by a mixing matrix

$$
V=V_{13} V_{12} V_{23}
$$

where $V_{i j}$ is a rotation matrix in between the $i, j$ rows and columns by an angle $\theta_{i j}$. For example

$$
V_{12}=\left(\begin{array}{ccc}
c_{12} & s_{12} & 0 \\
-s_{12} & c_{12} & 0 \\
0 & 0 & 1
\end{array}\right)
$$

where $s_{i j}=\sin \theta_{i j}$ and $c_{i j}=\cos \theta_{i j}$. We have taken the mixing matrix to be real for simplicity. Then the Majorana mass matrix in this basis is

$$
M_{N}=m_{D}^{\prime T} V m_{\mathrm{eff}}^{-1} V^{T} m_{D}^{\prime}
$$

The lepton asymmetry is created by the decay of the lightest of the heavy majorana neutrinos. Consequantly we have to go to a basis in which the Majorana mass matrix is diagonal. This can be diagonalized by a matrix $K$ so that

$$
M_{N}^{\text {diag }}=K^{T} M_{N} K
$$

Note the Dirac and Majorana mass matrices are related through Eqn (11). It can be easily seen that the Dirac neutrino mass matrix in such a basis is,

$$
m_{D}=K m_{D}^{\prime} K^{T}
$$

The CP-asymmetries in the neutrino decays arize from the interference between the tree level and one-loop level decay channels [13,14,

$$
\epsilon_{j}=-\frac{1}{8 \pi v_{2}^{2}} \frac{1}{\left(m_{D}^{\dagger} m_{D}\right)_{j j}} \sum_{n \neq j} \operatorname{Im}\left[\left(m_{D}^{\dagger} m_{D}\right)_{n j}^{2}\right] g\left(\frac{a_{n}}{a_{j}}\right),
$$


where

$$
g(x)=\sqrt{x}\left[\ln \left(\frac{1+x}{x}\right)+\frac{2}{x-1}\right]
$$

and $v_{2}=v \sin \beta$. The other parameter of most interest is the mass parameter

$$
\tilde{m}_{1}=\frac{\left(m_{D}^{\dagger} m_{D}\right)_{11}}{M_{1}}
$$

which largely controls the amount of dilution caused by the lepton number violating scattering f. A large enough lepton asymmetry can result only if $\tilde{m}_{1}$ is in the range $10^{-5}<\tilde{m}_{1}<10^{-2}\left[13\right.$. For too small values of $\tilde{m}_{1}$, the Yukawa interactions are too weak to bring the neutrinos into equilibrium at high temperatures. For too high $\tilde{m}_{1}$, the lepton number violating scatterings wash out most of the asymmetry after it is generated.

Scanning over the alloowed ranges for the neutrino mixing angles and taking $\lambda=0.22$, one can find the regions in the $m_{1}-m_{2}-m_{3}$ parameter space for which the two conditions are satisfied

- $\left|\epsilon_{1}\right|>10^{-6}$

- $10^{-5}<\tilde{m}_{1}<10^{-2}$

We assume for definiteness in our numerical results that the neutrino Dirac mass matrix is identical to the up quark mass matrix . There is an undertermined phase in this procedure which we can assume is such that the maximal CP-asymmetry is obtained since we are determining the points for which it is possible to obtain the required baryon asymmetry.

\footnotetext{
${ }^{4}$ This parameter is especially important in the supersymmetric scenarios there exists a large number of scattering diagrams which are lepton number violating, and the Yukawa interactions are much more important.

${ }^{5}$ Relaxing this choice, or choosing a different sign for the neutrino mixing angles will change the quantitative results, but not the qualitative ones.
} 
As an example the allowed regions for Texture 4 is shown in Fig. 1 with the neutrino mixings set to $s_{23}=0.55, s_{12}=0.07$ and $s_{13}=-0.03$. For a particular choice of the neutrino mixing angles only a narrow three dimension region is allowed in the full parameter space. This region is characterized by larger values of the lightest right-handed neutrino mass $\left(10^{7}<M_{1}<10^{8} \mathrm{GeV}\right)$, and smaller values for the neutrino quark mass matrix parameter $\left(m_{D}^{\dagger} m_{D}\right)_{11}$. This represents a moderately fine-tuned solution which can be understood from the hierarchical structure of the mass matrices as follows.

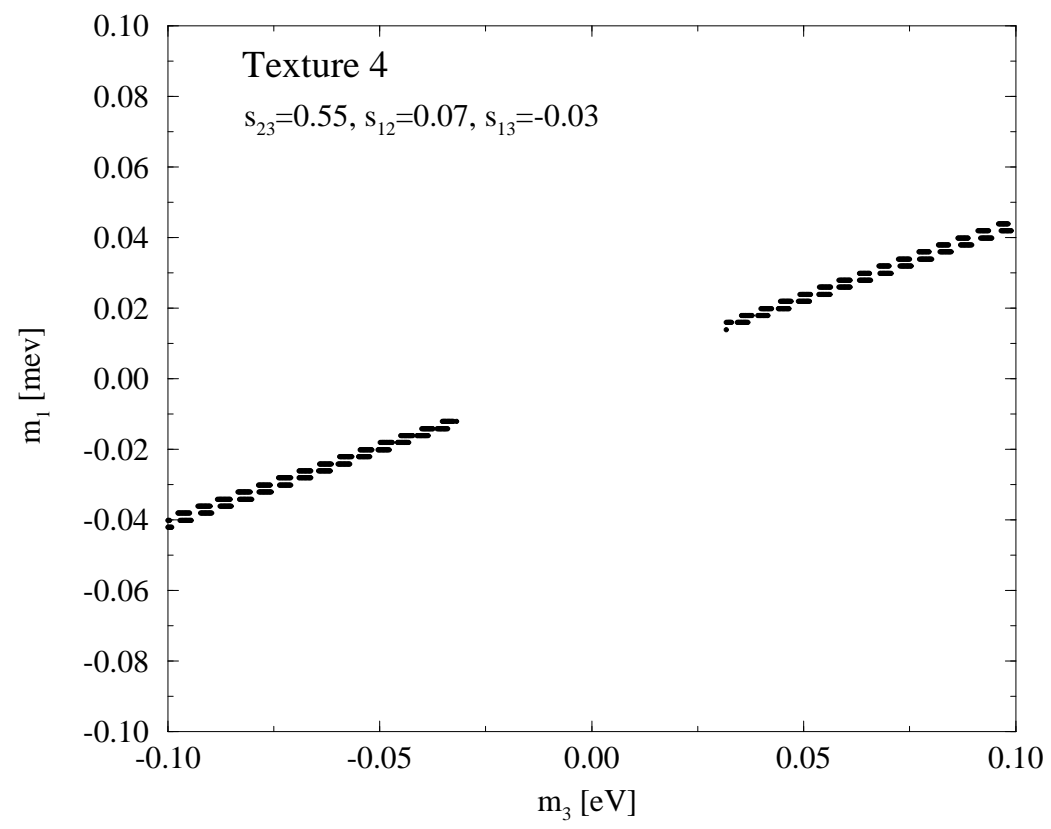

Fig. 1. The allowed solutions for $m_{1}$ and $m_{3}$ that generate a sufficient baryon asymmetry for Texture 4 .

Consider a generic matrix exhibiting the hierarchy given by the textures

$$
\lambda_{D}^{\text {generic }}=\left(\begin{array}{ccc}
A \lambda^{8} & B \lambda^{6} & C \lambda^{4} \\
B^{\prime} \lambda^{6} & D \lambda^{4} & E \lambda^{2} \\
C^{\prime} \lambda^{4} & E^{\prime} \lambda^{2} & 1
\end{array}\right),
$$

where $A, B, B^{\prime} \ldots E^{\prime}$ are coefficients of order one. The leading $\lambda$ dependence of the parameters most important to leptogenesis for this generic hierarchy is shown in Table [I]. Also shown are the $\lambda$ dependence of the five RRR textures; one sees that Textures 2, 4 and 5 have 
$\mathrm{CP}$ violation $\epsilon_{1}$ of the same order as the generic case, while Textures 1 and 3 are further suppressed by powers of $\lambda$ (the suppression by $\lambda^{4}$ assumes that the texture zeros are exact). Furthermore Texture 1 has a enhanced $\tilde{m}_{1}$ which implies that for this texture the dilution factor is very large.

\begin{tabular}{|c|c|c|c|c|}
\hline Texture & $\left(m_{D}^{\dagger} m_{D}\right)_{11}$ & $M_{1}$ & $\tilde{m}_{1}$ & $\epsilon_{1}$ \\
\hline generic & $\lambda^{8}$ & $\lambda^{16}$ & $\lambda^{-8}$ & $\lambda^{12}$ \\
\hline 1 & $\lambda^{12}$ & $\lambda^{16}$ & $\lambda^{-4}$ & $\lambda^{16}$ \\
\hline 2 & $\lambda^{8}$ & $\lambda^{16}$ & $\lambda^{-8}$ & $\lambda^{12}$ \\
\hline 3 & $\lambda^{8}$ & $\lambda^{16}$ & $\lambda^{-8}$ & $\lambda^{16}$ \\
\hline 4 & $\lambda^{8}$ & $\lambda^{16}$ & $\lambda^{-8}$ & $\lambda^{12}$ \\
\hline 5 & $\lambda^{8}$ & $\lambda^{16}$ & $\lambda^{-8}$ & $\lambda^{12}$ \\
\hline
\end{tabular}

TABLE II. The leading $\lambda$ dependence of various parameters important to leptogenesis. Textures 1 and 3 have leading $\epsilon_{1}$ that is subleading in $\lambda$, so any solutions are exceedingly fine-tuned. In addition, Texture 1 typically has too large a dilution factor because $\tilde{m}_{1}$ is enhanced. Only Textures 2, 4 and 5 offer solutions that are not overly fine-tuned. 
If one assumes the hierarchy suggested by the RRR textures then there must be some fine-tuning of the light neutrino masses to get a solution with a sufficient amount of leptogenesis. That is because the parameter $\tilde{m}_{1}$ which governs the dilution of the produced lepton asymmetry is too large for typical values of the light neutrino masses, related via the seesaw as

$$
\tilde{m}_{1} \sim \lambda^{-8} \frac{v_{2}^{2}}{M_{1}}
$$

The RRR textures fall into the category of the generic texture defined above, where the eigenvalues are in the ratio $1: \lambda^{-4}: \lambda^{-8}$. We find that in this case there is some fine-tuning required to achieve the required amount of leptogenesis; in Fig. 1 there is only a small pencillike region which produces an adequate lepton asymmetry. For other choices of the neutrino mixing angles there is a different linear correlation between the neutrino masses, but the same fine-tuning is required. This is because $\tilde{m}_{1}$ predicted by the RRR textures is typically too large by a factor $\lambda^{4}$ and the CP-violation $\epsilon_{1}$ is too small by the same factor. If instead a more modest hierarchy for the neutrino Dirac masses is assumed, say $1: \lambda^{-2}: \lambda^{-4}$, then one finds that a typical value of the light neutrino masses allowed by the solar and atmospheric neutrino experiments can generated the required level of leptogenesis. This suggests that the leptogenesis occurs more naturally in cases with the reduced hierarchy, and the RRR textures must be fine-tuned to achieve the required leptogenesis.

Since leptogenesis can only occur after the end of inflation, the subsequent thermal production of massive gravitinos can occur. The gravitinos interact weakly and the late decays of these can modify the observed abundances of light elements or overclose the universe [9]. The right-handed neutrino mass $M_{1}$ in our numerical solutions is sufficiently light to admit a solution to the gravitino problem [10]. 


\section{BOLTZMANN EQUATIONS}

The size of the lepton asymmetry that results can be calculated using the full set of Boltzmann equations [11]. These have been studied in the scenarios where there are only the Standard Model particles [12], but have become available recently in the full supersymmetric case as well [13]. We consider the supersymmetric case here since the supersymmetric Yukawa interactions are sufficient to produce a thermal population of right-handed neutrinos after reheating (the nonsupersymmetric model requires the introduction of new interactions [17]). The Boltzmann equations become quite involved for the supersymmetric case, where it is know that the dilution factor can be enhanced over the non-supersymmetric case because of the enhanced effect of the Yukawa interactions.

In principle what is desirable is to scan over all possible values for the masses and mixings of the neutrinos that are consistent with the solar and atmospheric neutrino oscillation experiments, and to determine the viable parameter choices. We do not do that here for three reasons: (1) computational power is exhausted after a few points, as each solution of the Boltzmann equation for a parameter choice involves numerically integrating a set of differential equations each of which involves a further numerical integration (this integration is needed to calculate the reaction density for the two body scatterings which can occur over the full kinematic range), (2) the exact equality between the neutrino and up quark Dirac matrices is probably only approximate, so our results must be considered qualitative only, and (3) there is an unknown phase in the Dirac neutrino mass matrix that controls the amount of $\mathrm{CP}$ violation in the heavy neutrino decays, so one can only determine an upper bound on the amount of lepton asymmetry generated. So we confine ourselves here to demonstrating that a particular parameter and texture choice can produce a baryon asymmetry consistent with observed result

$$
Y_{B}=\frac{n_{B}}{s}=(0.6-1) \times 10^{-10}
$$

where $n_{B}$ is the number density of baryons, and $s$ is the entropy density. This quantity con- 
veniently is insensitive to the dilution that comes about from the expansion of the universe. Similar densities $Y_{i}$ can be defined for all number densities $n_{i}$.

Figure 2 shows the evolution of the neutrino densities and the lepton asymmetry as a function of the temperature $T$ through $z=M_{1} / T$ for Texture 4 with neutrino masses of $m_{1}=1.5 \times 10^{-5} \mathrm{eV}, m_{2}=3.0 \times 10^{-3} \mathrm{eV}$ and $m_{3}=4.0 \times 10^{-2} \mathrm{eV}$. This point is one of the allowed solutions shown in Fig. 1 that satisfies the requirements for neutrino oscillations and for the requirements on $\epsilon_{1}$ and $\tilde{m}_{1}$. For these masses the right-handed Majorana mass is $M_{1}=2.9 \times 10^{7} \mathrm{GeV}$. Assuming a maximal CP-violating phase, the amount of CP-violation from the decays of the lightest Majorana neutrino is $\epsilon_{1}=-2.1 \times 10^{-6}$. These masses are consistent with the constraints from the solar and atmospheric neutrino experiments in Eq. 6 . The evolution of the densities proceeds to the right as the temperature of the universe decreases. The figure shows the equilibrium density of the lightest Majarona neutrino $Y_{N_{1}}^{\text {eq }}$ along with the computed density $Y_{N_{1}}$. Nonzero asymmetries of lepton number from fermions $Y_{L_{f}}$ and from scalars $Y_{L_{s}}$ develop, and change sign (hence the dip in the figure), and finally asymptote to a constants for values of $z=M_{1} / T>3-4$. Sscattering processes involving exchange of supersymmetric particles enforce that $Y_{L_{f}} \approx Y_{L_{s}}$. Finally, for completeness, we show the scalar neutrino asymmetry for the supersymmetric partner to the lightest Majorana neutrino $Y_{1-}=Y_{\tilde{N}_{1}^{c}}-Y_{\tilde{N}_{1}^{c} \dagger}$. This asymmetry also changes sign before eventually vanishing for large values of $z$. The total density of scalar neutrinos $Y_{1+}=Y_{\tilde{N}_{1}^{c}}+Y_{\tilde{N}_{1}^{c} \dagger}$ is indistinguishable from $Y_{N_{1}}$ and is omitted from the figure. 


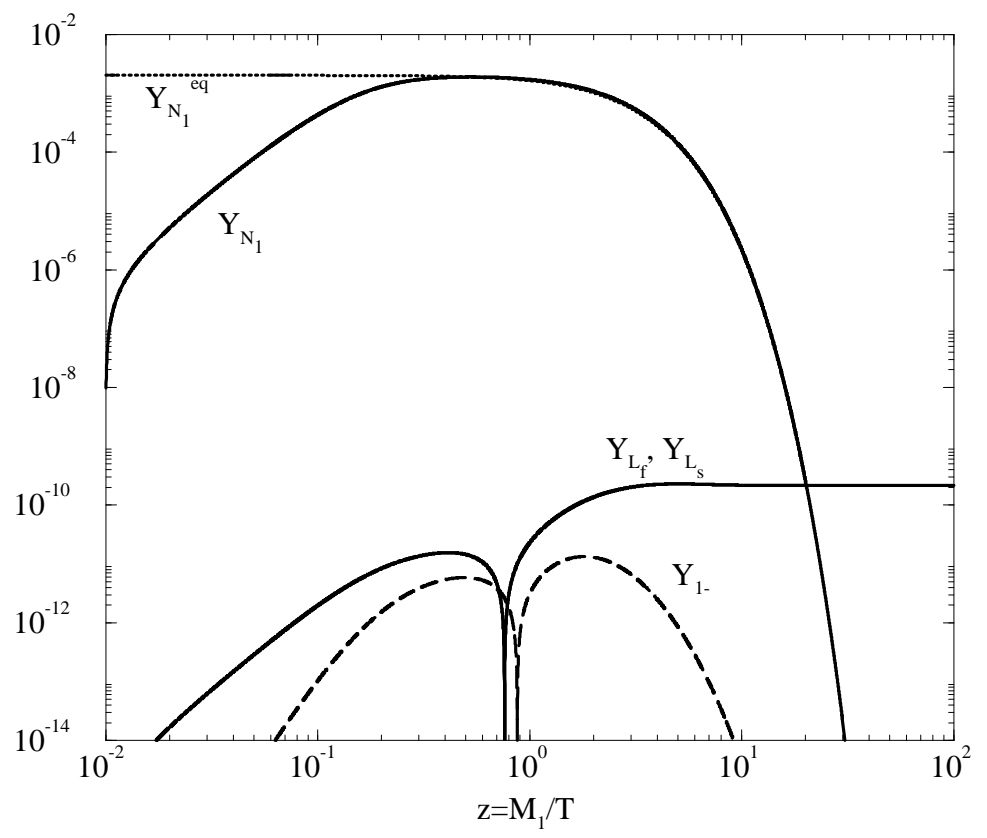

Fig. 2. The evolution of the fermionic and baryonic lepton asymmetries. The asymmetries $Y_{L_{f}}$ and $Y_{L_{s}}$ asymptote to a constant value which is recycled into a baryon asymmetry that is sufficient to account for experiment if $Y_{L_{f}}=Y_{L_{s}}=$ $-(0.9-1.4) \times 10^{-10}$.

The baryon asymmetry is related to the lepton asymmetry (in the supersymmetric case) via

$$
Y_{B}=-\frac{8}{15} Y_{L}
$$

so the observed baryon asymmetry is generated provided that the asymptotic value (for small $T$ ) of the lepton asymmetry is

$$
Y_{L_{f}}=Y_{L_{s}}=-(0.6-0.9) \times 10^{-10}
$$

So Fig. 2 shows a consistent solution of Texture 4 that explains the baryon asymmetry of the universe which is arising after a fine-tuning of the neutrino masses. 


\section{CONCLUSION}

We studied the possibility that the baryon asymmetry of the universe could result from lepton number violating decays of heavy Majorana neutrinos. We assumed the Dirac neutrino texture was given by the set of Ramond-Roberts-Ross textures with five zeros (which gives rise to correct masses and mixings of the charged fermions). The heavy Majonana neutrino mass matrix is obtained by inverting the type-I see-saw formula where the contributions from the left handed triplet Higgs are neglected, and using the neutrino masses and mixings required by the solar and atmospheric neutrino oscillation experiments. The lepton asymmetry is produced due to the lepton number violating decay of the lightest right handed neutrino. Contrary to naive expectations, the lightest eigenvalue of the heavy Majorana neutrino mass matrix is in the range $10^{5}-10^{7} \mathrm{GeV}$ even though the right handed gauge symmetry breaks at $M_{X}=10^{16} \mathrm{GeV}$. This is due to the hierarchy of the Dirac-type neutrino texture. We obtained the following results for the feasibility for each texture for generating the required baryon asymmetry: (a) A generic neutrino Dirac mass matrix with eigenvalues in the ratio $1: \lambda^{-4}: \lambda^{-8}$ can produce the observed baryon asymmetry via the baryogenesis via leptogenesis scenario in narrow ranges of light neutrino masses. This predicts a strong correlation between the light neutrino masses, but only because the masses of the neutrinos must be carefully tuned to achieve the required magnitude of leptogenesis. A neuriino Dirac mass matrix eigenvalues in the ratio $1: \lambda^{-2}: \lambda^{-4}$ naturally gives a lepton asymmetry of the required level. (b) Textures 2,4,5 generate the amount of leptogenesis expected in models with a neutrino Dirac mass hierarchy with eigenvalues in the ratio $1: \lambda^{-4}: \lambda^{-8}$. (b) The position of the texture zeros in Textures 1 and 3 result in a further suppression of the generated lepton asymmetry.

We carried out a detailed analysis of the generated baryon asymmetry by solving the Boltzmann equations for a supersymmetric model numerically for Texture 4. This demonstrates that the required texture can be compatible with baryogenesis via leptogenesis for some specific values of the light neutrino masses consistent with observations in solar and 
atmospheric neutrino experiments. 


\section{REFERENCES}

[1] M. Fukugita and T. Yanagida, Phys. Lett. 174, 45 (1986); Recent review can be found at, A. Pilaftsis, CERN-TH-98-386, hep-ph/9812256.

[2] A. D. Sakharov, Zh. Eksp. Teor. Fiz. Pis'ma 5, 32 (1967); JETP Lett. 91B, 24 (1967).

[3] O. Bertolami, D. Colladay, V. A. Kostelecký and R. Potting, Phys. Lett. B395, 178 (1997).

[4] See for example, G. R. Farrar, M. E. Shaposhnikov Phys. Rev. Lett. 702833 (1993), Erratum-ibid. 71 210, 1993; Phys. Rev. D 50 , 774 (1994).

[5] J. M. Cline, talk given at Conference on Strong and Electroweak Matter (SEWM 98), Copenhagen, Denmark, 2-5, Dec 1998, hep-ph/9902328, and references therein.

[6] K. S. Hirata et al, Phys. Lett. B205, 416 (1988); Phys. Lett. B280, 146 (1992); R. Becker-Szendy et. al, Phys. Rev. D46, 3120 (1992); M. Goodman et. al., Nucl. Phys. B38. (Proc. Supp.), 337 (1995). Y. Suzuki, Talk presented at Neutrino 98, Takayama, Japan, June 1998.

[7] G. K. Leontaris, S. Lola, C. Scheich and J. D. Vergados, Phys. Rev. D53, 6381 (1996).

[8] P. Ramond, R. G. Roberts and G. G. Ross, Nucl. Phys. B406, 19 (1993).

[9] M. Yu. Klopov and A. D. Linde, Phys. Lett. B138, 265 (1984); J. Ellis, J. Kim and D. V. Nanopoulos, Phys. Lett. B145, 181 (1984).

[10] M. Bolz, W. Buchmüller and M. Plümacher, Phys. Lett. B443, 209 (1998); D. Delepine and U. Sarkar, hep-ph/9811479.

[11] J. N. Fry, K. A. Olive and M. S. Turner; Phys. Rev. D22, 2953 (1980), Phys.Rev. D 22, 2977 (1980).

[12] M. Luty, Phys. Rev. D45, 455 (1992). 
[13] W. Buchmüller and M. Plümacher, Phys. Lett. B389, 73 (1996); Phys. Lett. B431, 354 (1998), M. Plümacher, hep-ph/9807557.

[14] L. Covi, E. Roulet and F. Vissani, Phys. Lett. B384, 169 (1996); M. Flanz, E. A. Paschos, U. Sarkar, J. Weiss, Phys. Lett. B389 693 (1996); R. Rangarajan, U. Sarkar, Phys. Lett. B442, 243 (1998).

[15] N. Hata and P. Langacker, Phys. Rev. D56, 6107 (1997).

[16] W. Buchmüller and T. Yanagida, Phys. Lett. B445, 399 (1999).

[17] M. Plümacher, Z. Phys. C74, 549 (1997). 